\title{
PAIRS OF PATHS AND CRITICAL POINTS
}

\section{FLORIN CARAGIU and IOANA CARAGIU}

(Received 22 May 2000)

\begin{abstract}
Two sufficient conditions are presented, in terms of the values taken by a holomorphic function $f(z)$ on a pair of smooth paths intersecting at a point $z_{0}$ in its domain, implying that $f^{\prime}\left(z_{0}\right)=0$.
\end{abstract}

2000 Mathematics Subject Classification. 30D20, 30C15.

In the present paper, we present two sufficient conditions expressed in terms of the values taken by a holomorphic function $f$ on a pair of smooth paths intersecting at a point $z_{0}$ in the domain of $f$, with tangent vectors at $z_{0}$ linearly independent over $\mathbb{R}$, implying that $f^{\prime}\left(z_{0}\right)=0$.

THEOREM 1. Let $f: D \subset \mathbb{C} \rightarrow \mathbb{C}$ be a holomorphic function, where $D \subset \mathbb{C}$ is a domain and let $\gamma, \Gamma:(0,1) \rightarrow D$ be two smooth $\left(C^{1}\right)$ paths. Assume the following:

(i) for a certain $z_{0} \in D$ and some $t_{1}, t_{2} \in(0,1)$ we have $z_{0}=\gamma\left(t_{1}\right)=\Gamma\left(t_{2}\right)$;

(ii) $\gamma^{\prime}\left(t_{1}\right)$ and $\Gamma^{\prime}\left(t_{2}\right)$ linearly independent over $\mathbb{R}$ (i.e., non-collinear),

(iii) $|f(z)|$ takes a constant value on the subset $\gamma((0,1)) \cup \Gamma((0,1))$ of $D$. Then $f^{\prime}\left(z_{0}\right)=0$.

Proof. Let $f=u+i v, \gamma=\gamma_{1}+i \gamma_{2}$, and $\Gamma=\Gamma_{1}+i \Gamma_{2}$, where $u, v$ are real-valued functions while $\gamma_{1}, \gamma_{2}, \Gamma_{1}, \Gamma_{2}$ are real-valued smooth paths. The assumption (iii) can be written as

$$
u^{2}(\gamma(t))+v^{2}(\gamma(t))=u^{2}(\Gamma(t))+v^{2}(\Gamma(t))=c
$$

for any $t \in(0,1)$, where $c$ is some constant. Note first that if $c=0$, from (1) together with the identity theorem of the holomorphic functions it follows that $f(z)=0$ for any $z \in D$. This being the case, we assume $c \neq 0$ from now on. We differentiate (1) with respect to $t$. We then have, for any $t \in(0,1)$,

$$
\frac{d}{d t}\left(u^{2}(\gamma(t))+v^{2}(\gamma(t))\right)=0
$$

that is, by using the chain rule,

$$
\begin{aligned}
2 u(\gamma(t)) u_{x}(\gamma(t)) \gamma_{1}^{\prime}(t)+2 u(\gamma(t)) u_{y}(\gamma(t)) \gamma_{2}^{\prime}(t) \\
+2 v(\gamma(t)) v_{x}(\gamma(t)) \gamma_{1}^{\prime}(t)+2 v(\gamma(t)) v_{y}(\gamma(t)) \gamma_{2}^{\prime}(t)=0
\end{aligned}
$$

together with the similar relation for $\Gamma$ :

$$
\begin{aligned}
2 u(\Gamma(t)) u_{x}(\Gamma(t)) \Gamma_{1}^{\prime}(t)+2 u(\Gamma(t)) u_{y}(\Gamma(t)) \Gamma_{2}^{\prime}(t) \\
+2 v(\Gamma(t)) v_{x}(\Gamma(t)) \Gamma_{1}^{\prime}(t)+2 v(\Gamma(t)) v_{y}(\Gamma(t)) \Gamma_{2}^{\prime}(t)=0
\end{aligned}
$$


holding also for any $t \in(0,1)$. By using the Cauchy-Riemann equations in (3) and (4), respectively, we get, after a convenient grouping of terms,

$$
\begin{aligned}
& u(\gamma(t))\left[u_{x}(\gamma(t)) \gamma_{1}^{\prime}(t)-v_{x}(\gamma(t)) \gamma_{2}^{\prime}(t)\right]+v(\gamma(t))\left[u_{x}(\gamma(t)) \gamma_{2}^{\prime}(t)+v_{x}(\gamma(t)) \gamma_{1}^{\prime}(t)\right]=0, \\
& u(\Gamma(t))\left[u_{x}(\Gamma(t)) \Gamma_{1}^{\prime}(t)-v_{x}(\Gamma(t)) \Gamma_{2}^{\prime}(t)\right]+v(\Gamma(t))\left[u_{x}(\Gamma(t)) \Gamma_{2}^{\prime}(t)+v_{x}(\Gamma(t)) \Gamma_{1}^{\prime}(t)\right]=0,
\end{aligned}
$$

for any $t \in(0,1)$. By specializing $t=t_{1}$ in (5) and $t=t_{2}$ in (6), we obtain

$$
\begin{aligned}
& u\left(z_{0}\right)\left[u_{x}\left(z_{0}\right) \gamma_{1}^{\prime}\left(t_{1}\right)-v_{x}\left(z_{0}\right) \gamma_{2}^{\prime}\left(t_{1}\right)\right]+v\left(z_{0}\right)\left[u_{x}\left(z_{0}\right) \gamma_{2}^{\prime}\left(t_{1}\right)+v_{x}\left(z_{0}\right) \gamma_{1}^{\prime}\left(t_{1}\right)\right]=0, \\
& u\left(z_{0}\right)\left[u_{x}\left(z_{0}\right) \Gamma_{1}^{\prime}\left(t_{2}\right)-v_{x}\left(z_{0}\right) \Gamma_{2}^{\prime}\left(t_{2}\right)\right]+v\left(z_{0}\right)\left[u_{x}\left(z_{0}\right) \Gamma_{2}^{\prime}\left(t_{2}\right)+v_{x}\left(z_{0}\right) \gamma_{1}^{\prime}\left(t_{2}\right)\right]=0 .
\end{aligned}
$$

Since $u^{2}\left(z_{0}\right)+v^{2}\left(z_{0}\right)=c \neq 0$, it follows from (7) that

$$
\left(u\left(z_{0}\right), v\left(z_{0}\right)\right) \neq(0,0)
$$

is a nontrivial solution of the linear homogeneous system

$$
\begin{gathered}
X\left[u_{x}\left(z_{0}\right) \gamma_{1}^{\prime}\left(t_{1}\right)-v_{x}\left(z_{0}\right) \gamma_{2}^{\prime}\left(t_{1}\right)\right]+Y\left[u_{x}\left(z_{0}\right) \gamma_{2}^{\prime}\left(t_{1}\right)+v_{x}\left(z_{0}\right) \gamma_{1}^{\prime}\left(t_{1}\right)\right]=0, \\
X\left[u_{x}\left(z_{0}\right) \Gamma_{1}^{\prime}\left(t_{2}\right)-v_{x}\left(z_{0}\right) \Gamma_{2}^{\prime}\left(t_{2}\right)\right]+Y\left[u_{x}\left(z_{0}\right) \Gamma_{2}^{\prime}\left(t_{2}\right)+v_{x}\left(z_{0}\right) \gamma_{1}^{\prime}\left(t_{2}\right)\right]=0,
\end{gathered}
$$

and so

$$
\left|\begin{array}{cc}
u_{x}\left(z_{0}\right) \gamma_{1}^{\prime}\left(t_{1}\right)-v_{x}\left(z_{0}\right) \gamma_{2}^{\prime}\left(t_{1}\right) & u_{x}\left(z_{0}\right) \gamma_{2}^{\prime}\left(t_{1}\right)+v_{x}\left(z_{0}\right) \gamma_{1}^{\prime}\left(t_{1}\right) \\
u_{x}\left(z_{0}\right) \Gamma_{1}^{\prime}\left(t_{2}\right)-v_{x}\left(z_{0}\right) \Gamma_{2}^{\prime}\left(t_{2}\right) & u_{x}\left(z_{0}\right) \Gamma_{2}^{\prime}\left(t_{2}\right)+v_{x}\left(z_{0}\right) \gamma_{1}^{\prime}\left(t_{2}\right)
\end{array}\right|=0 .
$$

By expanding the determinant, equation (10) can be rewritten as

$$
\left(u_{x}^{2}\left(z_{0}\right)+v_{x}^{2}\left(z_{0}\right)\right)\left(\gamma_{1}^{\prime}\left(t_{1}\right) \Gamma_{2}^{\prime}\left(t_{2}\right)-\Gamma_{1}^{\prime}\left(t_{2}\right) \gamma_{2}^{\prime}\left(t_{1}\right)\right)=0 .
$$

On the other hand, the assumption (iii) can be rewritten as

$$
\left|\begin{array}{cc}
\gamma_{1}^{\prime}\left(t_{1}\right) & \gamma_{2}^{\prime}\left(t_{1}\right) \\
\Gamma_{1}^{\prime}\left(t_{2}\right) & \Gamma_{2}^{\prime}\left(t_{2}\right)
\end{array}\right| \neq 0 .
$$

Finally, from (11) and (12) it follows that

$$
u_{x}^{2}\left(z_{0}\right)+v_{x}^{2}\left(z_{0}\right)=0
$$

that is, $u_{x}\left(z_{0}\right)=v_{x}\left(z_{0}\right)=0$. This, together with the Cauchy-Riemann relations [1] implies $u_{y}\left(z_{0}\right)=v_{x}\left(z_{0}\right)=0$ and so $f^{\prime}\left(z_{0}\right)=0$. This concludes the proof of Theorem 1 .

The following exercise represents an interesting corollary of Theorem 1.

COROLlary 2. Let $D \subset \mathbb{C}$ be a domain which contains the square $[-1,1] \times[-1,1]$. Assume that $f: D \rightarrow \mathbb{C}$ is a holomorphic function with the property that there exists $c \in \mathbb{R}_{+}^{*}$ such that

$$
|f(x+i 0)|=c=\left|f\left(x+i \sin \left(\frac{1}{x}\right)\right)\right|
$$

for any $x \in(0,1)$. Then $f$ is a constant function. 
Proof. Let $\gamma, \Gamma:(0,1) \rightarrow \mathbb{C}$ defined by

$$
\gamma(t)=(t, 0), \quad \Gamma(t)=\left(t, \sin \left(\frac{1}{t}\right)\right),
$$

respectively. We have

$$
\gamma^{\prime}(t)=(1,0), \quad \Gamma^{\prime}(t)=\left(1,-\frac{1}{t^{2}} \cos \left(\frac{1}{t}\right)\right),
$$

for any $t \in(0,1)$. Consider the sequence

$$
t_{k}=\frac{1}{k \pi} \in(0,1)
$$

convergent to 0 . This choice of the sequence makes sure that

$$
\gamma\left(t_{k}\right)=\Gamma\left(t_{k}\right)=\left(t_{k}, 0\right)
$$

for any $k \geq 1$. We also have $\gamma^{\prime}\left(t_{k}\right)=(1,0)$ and $\Gamma^{\prime}\left(t_{k}\right)=\left(1,-k^{2}(-1)^{k} \pi^{2}\right)$ which implies immediately that $\gamma\left(t_{k}\right)$ and $\Gamma\left(t_{k}\right)$ are linearly independent over $\mathbb{R}$ for any $k \geq 1$. By Theorem 1,

$$
f^{\prime}\left(t_{k}+i 0\right)=0
$$

holds true for any $k \geq 1$. Since $f^{\prime}$ is holomorphic and $t_{k} \rightarrow 0 \in D(z=0 \in D$ is an accumulation point for the zeros of $f^{\prime}$ ), it follows that $f^{\prime}(z)=0$ for any $z \in D$, that is, $f$ is a constant on $D$.

Another result of similar flavour is the following theorem.

THEOREM 3. Let $f: \mathbb{C} \rightarrow \mathbb{C}$ be holomorphic on an open neighborhood $V$ of $z_{0}$, and let $\gamma_{1}, \gamma_{2}:(0,1) \rightarrow V$ be a pair of $C^{1}$ paths such that for some $t_{1}, t_{2} \in(0,1)$, we have $\gamma_{1}\left(t_{1}\right)=\gamma_{2}\left(t_{2}\right)=z_{0}$ and $\gamma_{1}^{\prime}\left(t_{1}\right), \gamma_{2}^{\prime}\left(t_{2}\right)$ are linearly independent over $\mathbb{R}$. We also assume that $f\left(\gamma_{k}(t)\right) \in \mathbb{R}, k=1,2$ for any $t \in(0,1)$. Then, under the above assumptions, $f^{\prime}\left(z_{0}\right)=0$. If, in addition, $\arg \left(\gamma_{1}^{\prime}\right), \arg \left(\gamma_{2}^{\prime}\right)$ are constant functions, then there exists a nonnegative integer $n$ and a holomorphic function $h$ defined on some open neighborhood of 0 such that $f(z)=h\left(\left(z-z_{0}\right)^{n}\right)$ for $z \in V$.

Proof. Let $\phi$ be the angle between $\gamma_{1}^{\prime}\left(t_{1}\right)$ and $\gamma_{2}^{\prime}\left(t_{2}\right)$. Consider two sequences $\left\{x_{n}\right\},\left\{y_{n}\right\}$ of numbers from $(0,1)$ such that $\lim _{n \rightarrow \infty} x_{n}=t_{1}$ while $\lim _{n \rightarrow \infty} y_{n}=t_{2}$. Then

$$
\begin{aligned}
f^{\prime}\left(z_{0}\right) & =\lim _{n \rightarrow \infty} \frac{f\left(\gamma_{1}\left(x_{n}\right)\right)-f\left(\gamma_{1}\left(t_{1}\right)\right)}{\gamma_{1}\left(x_{n}\right)-\gamma_{1}\left(t_{1}\right)} \\
& =\lim _{n \rightarrow \infty} \frac{\left(f\left(\gamma_{1}\left(x_{n}\right)\right)-f\left(\gamma_{1}\left(t_{1}\right)\right)\right) /\left(x_{n}-t_{1}\right)}{\left(\gamma_{1}\left(x_{n}\right)-\gamma_{1}\left(t_{1}\right)\right) /\left(x_{n}-t_{1}\right)} \in \mathbb{R} e^{-i \arg \left(\gamma_{1}^{\prime}\left(t_{1}\right)\right)} .
\end{aligned}
$$

In a similar way, it is shown that

$$
f^{\prime}\left(z_{0}\right) \in \mathbb{R} e^{-i \arg \left(\gamma_{2}^{\prime}\left(t_{2}\right)\right)} .
$$

From (20) and (21), together with the assumption that $\gamma_{1}^{\prime}\left(t_{1}\right)$ and $\gamma_{2}^{\prime}\left(t_{2}\right)$ are linearly independent over $\mathbb{R}$, it follows that $f^{\prime}\left(z_{0}\right)$ has to be zero. This concludes the proof of the 
first part of the theorem. We assume now that $\arg \left(\gamma_{1}^{\prime}\right), \arg \left(\gamma_{2}^{\prime}\right)$ are constant functions, say $\arg \left(\gamma_{k}^{\prime}\right)=c_{k}, k=1,2$, where $c_{1} \neq c_{2}$. Then, keeping in mind that $f\left(\gamma_{k}(t)\right) \in \mathbb{R}$, $k=1,2$ for any $t \in(0,1)$, we see that

$$
f^{\prime}\left(\gamma_{k}(t)\right) \in \mathbb{R} e^{-i c_{k}}
$$

for $k=1,2$ and $t \in(0,1)$. By induction on $r$, we can show that

$$
f^{(r)}\left(\gamma_{k}(t)\right) \in \mathbb{R} e^{-i r c_{k}}
$$

holds true for any nonnegative integer $r$ where $k=1,2$ and $t \in(0,1)$. Indeed, for $r=0$ and $r=1$, equation (23) is already shown. Assuming that (23) is true, by differentiation we get

$$
f^{(r+1)}\left(\gamma_{k}(t)\right) \gamma_{k}^{\prime}(t) \in \mathbb{R} e^{-i r c_{k}} .
$$

From (24) and the fact that $\arg \left(\gamma_{k}^{\prime}(t)\right)=c_{k}$, it follows that

$$
f^{(r+1)}\left(\gamma_{k}(t)\right) \in \mathbb{R} e^{-i(r+1) c_{k}}
$$

which concludes the inductive proof of (23). By specializing $t=t_{1}$ and then $t=t_{2}$ in (23), it follows that

$$
f^{(r)}\left(z_{0}\right) \in \mathbb{R} e^{-i r c_{1}} \cap \mathbb{R} e^{-i r c_{2}}
$$

for any $r=0,1,2, \ldots$. From (26) it follows that, for any given $r$, either $f^{(r)}\left(z_{0}\right)=0$ or $e^{i r \phi} \in \mathbb{R}$ (i.e., $r \phi \in 2 \pi \mathbb{Z}$ ). At this moment we distinguish two cases. First, if $\phi / \pi \in$ $\mathbb{R} \backslash \mathbb{Q}$, it follows that $f^{(r)}\left(z_{0}\right)=0$ for any $r=0,1,2, \ldots$ which implies that $f(z)$ is constant on a neighborhood of $z_{0}$ and this being the case the choice $h=$ constant $=c$ would work. We consider now the second case, when $\phi=m \pi / n$, where $0<m<n$, $m, n \in \mathbb{Z}_{>0},(m, n)=1$. From (26) it follows that $f^{(r)}\left(z_{0}\right)=0$ for any $r$ which is not divisible by $n$, since in this case $e^{i r \phi}=e^{i r m \pi / n} \notin \mathbb{R}$. Therefore, on some neighborhood of $z_{0}$ the power series expansion of $f$ has the form

$$
f(z)=\sum_{l \leq 0} a_{\ln }\left(z-z_{0}\right)^{\ln }=\sum_{l \geq 0} a_{\ln }\left[\left(z-z_{0}\right)^{n}\right]^{l} .
$$

If we denote

$$
h(z):=\sum_{l \geq 0} a_{l n} z^{l}
$$

it follows that $h$ is holomorphic on some neighborhood of 0 and satisfies $f(z)=$ $h\left(\left(z-z_{0}\right)^{n}\right)$. This concludes the proof of Theorem 3 .

\section{REFERENCES}

[1] L. V. Ahlfors, Complex Analysis. An Introduction to the Theory of Analytic Functions of one Complex Variable, International Series in Pure and Applied Mathematics, McGrawHill, New York, 1953. MR 14,857a. Zbl 052.07002.

Florin Caragiu and IoAna Caragiu: Department of Mathematics II, University POLITEHNiCA OF BUCHAREST, SPLAIUl INDEPENDENTEI 313, 79590 BUCHAREST, ROMANIA

E-mail address: m-caragiu1@onu . edu 


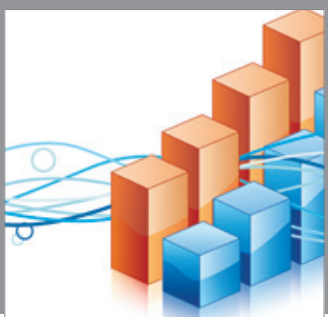

Advances in

Operations Research

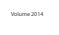

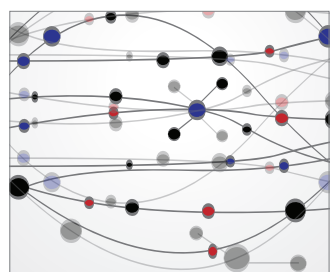

\section{The Scientific} World Journal
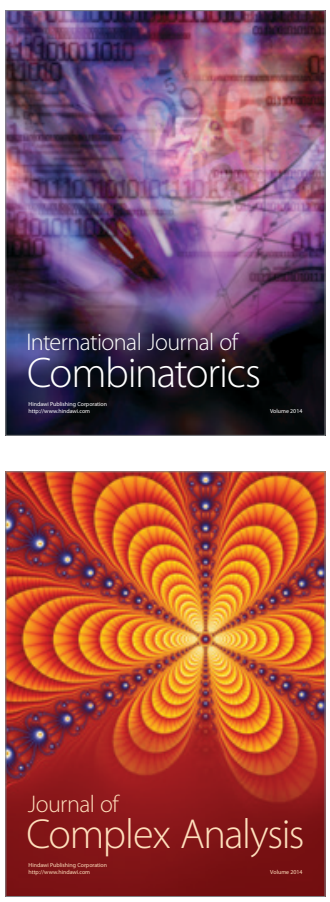

International Journal of

Mathematics and

Mathematical

Sciences
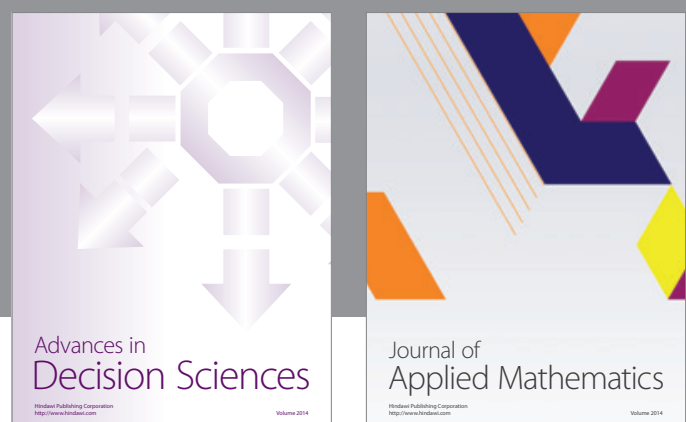

Journal of

Applied Mathematics
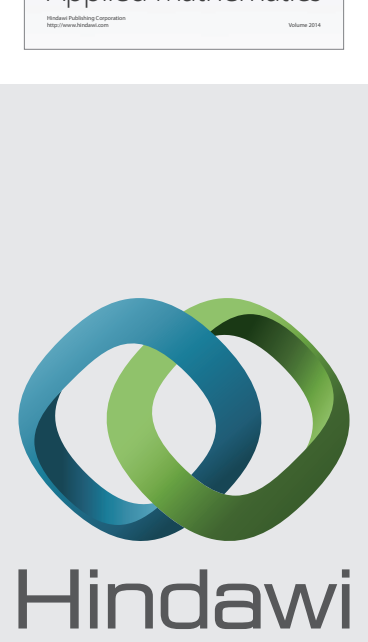

Submit your manuscripts at http://www.hindawi.com
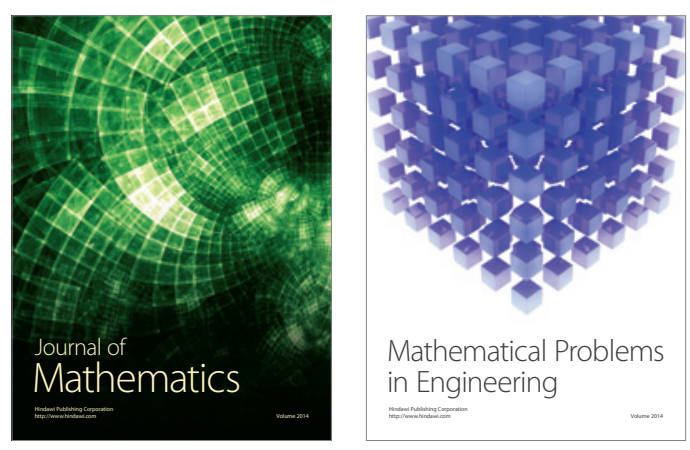

Mathematical Problems in Engineering
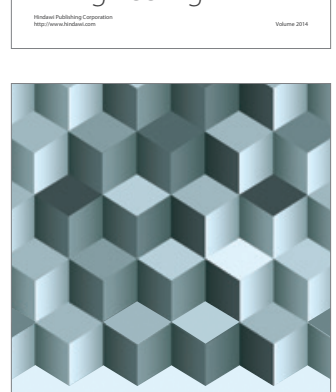

Journal of

Function Spaces
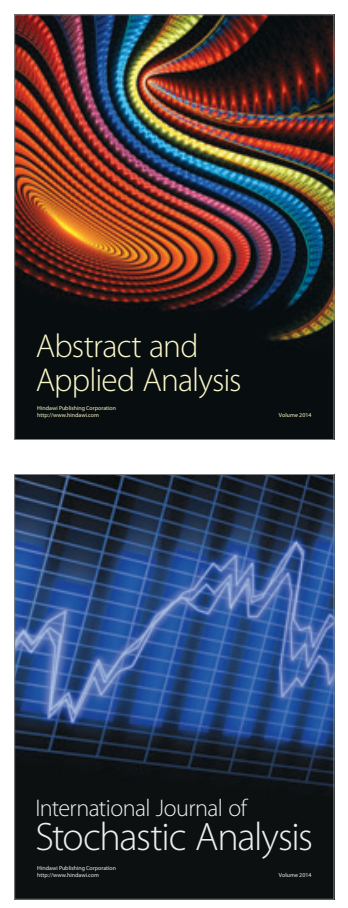

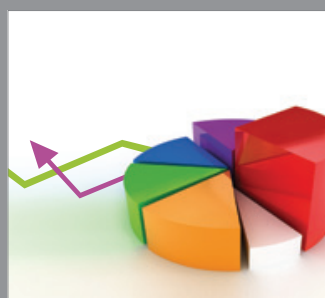

ournal of

Probability and Statistics

Promensencen
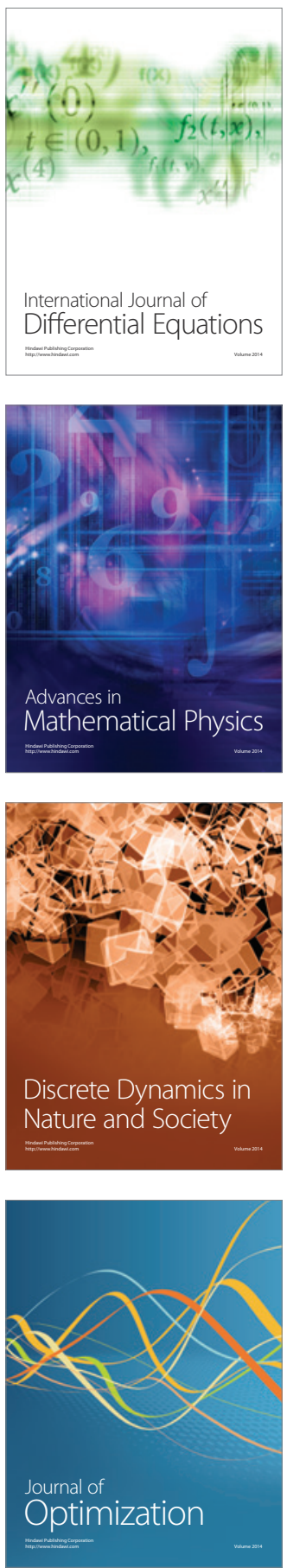